\title{
Application of Improved Stereographic Projection Method to Quantify Lithospheric Plate Motion Trajectory
}

\author{
Zhongju WEI and Fangfang $\mathrm{YU}^{1}$ \\ School of Mining and Civil Engineering, Liupanshui Normal University, Liupansuhi \\ 553004, Guizhou, China
}

\begin{abstract}
The traditional method of studying plate motion still cannot be used to obtain plate motion trajectory quantitatively. In this paper, we proposed a new method to quantitative determine plate motion trajectory. Depending on the paleomagnetic data of lithosphere plate and the stereographic projection principle. We selected the Wulff net as the basic projection net, improved and transformed the traditional stereographic projection methods. Projecting the paleomagnetic data (magnetic declination, palaeolatitude and geomagnetic pole coordinate) of the lithosphere plate into the improved stereographic projection net, we can get the analysis results of lithosphere plate stereographic projection. In our study, we took the Indian plate as an example, projected the paleomagnetic data (from Cretaceous) into the stereographic projection net, got the analysis results of motion trajectory of the Indian plate from Cretaceous. This method can be applied to quantify lithospheric plate motion trajectory.
\end{abstract}

Keywords. Improved stereographic projection, lithospheric plate, motion trajectory, paleomagnetic data, the Indian plate

\section{Introduction}

Following the theory of continental drift and sea floor spreading [1], the plate tectonics theory occurred last century which linked to advancements in geology. The plate tectonics theory explains movement of the Earth's lithosphere which is the earth's crust and the upper part of mantle. The lithosphere is divided into several plates [2], drift on the above of asthenosphere, these huge plates mutual discrete separation, or gather concentration, caused the continental drift and ocean opened and closed, inspired magmatic activity and large-scale geological disasters as earthquake activity, formed the various structural elements of the earth's surface, created conditions for the formation of mineral resources. Therefore, it is necessary to study the motion law of lithospheric plates.

Paleomagnetism which emerged in $1960 \mathrm{~s}$, provided strong evidence for continental drift and the sea floor, and greatly promoted the establishment of the theory of plate tectonics [3]. Since the establishment of the plate tectonics theory, the study of paleomagnetism has attracted the attention of geoscientists. Previous studies have

${ }^{1}$ Corresponding Author, Fangfang YU, School of Mining and Civil Engineering, Liupanshui Normal University, Liupansuhi 553004, Guizhou, China; Email: weizj2010ck@163.com. 
shown that the earth has a magnetic field [4]. In the process of rock formation or after rock formation, the magnetic materials contained in the rock were magnetized by the geomagnetic field, so that the rock can obtain natural residual magnetism. Like "fossils", the geomagnetic field directions in the rock were recorded [5]. Then, according to the theory that the magnetic field of the earth in geological history was an axial dipole field (GAD Hypothesis). During the same geological period, the paleomagnetic pole direction of each plate should be consistent. However, many palaeomagnetic measurement results of lithospheric plates shown that different magnetic pole directions had dispersity obviously, this is the result of the mutual movement between continental blocks. Through decades of efforts of paleomagnetic from various countries, the major global lithospheric plates movement and relative motion between blocks in the geological historical period were determined by using the paleomagnetic data. Fruitful results had been achieved, mainly including: a representative pole shift curve of the major plates of Africa, North America, Eurasia and India since $200 \mathrm{Ma}$ had been established for the study of neotectonics and regional tectonic evolution since Mesozoic [6], the geomagnetic polarity chronology had been established since 160Ma, which was widely used in the study of the regional neotectonic movement and the regional environmental evolution of the late Cenozoic [7], the paleomagnetic data were used to determine the movement and interplate interaction of the lithospheric plates at different levels in China [8-9]. However, previous studies only can determine the rotation of north-south motion components and clockwise (counter-clockwise) rotation, the traditional methods of quantitatively determining the movement of lithospheric plates using paleomagnetic data had shortcomings obviously.

In our study, we mentioned a new method that the motion of lithosphere plates can be completely quantified interpretation. We applied the stereographic projection method to the study of plate motion trajectory, improved and transformed the traditional stereographic projection method. Then, we took the Indian plate as an example, projected the paleomagnetic data (from the Cretaceous to the present) of Indian plate into the improved stereographic projection net, got the analysis results of motion trajectory of the Indian plate from Cretaceous.

\section{Principle of Stereographic Projection}

The stereographic projection is a drawing method to reflect the three-dimensional spatial geometry problems on the projection plane for research and processing, can visually express the straight and plane directions of three-dimensional space. It is easy to solve the angular distance problems between straight lines, straight lines and planes, planes in three-dimensional space. This method was first used in crystallography in 1902, and gradually extended to astronomy, navigation, surveying, geography, and geological sciences. At present, the stereographic projection method is mainly used to solve many practical problems in geometry and stress analysis of geological structure in geology.

\subsection{Spherical Projection}

Spherical projection is the intersection between straight lines, planes, and projection sphere surface in the three dimensional space. In the spherical projection coordinate 
system, the spherical projection can be directly determined according to the extension of the straight lines and planes in the three-dimensional space. In the spherical projection system, the spherical center of the projection ball is called the origin (point $\mathrm{O}$ in figure 1). The horizontal plane passing through the origin is called the equatorial plane (plane NESW in figure 1), which divides the projection ball into upper hemisphere and lower hemisphere. The great circle that the equatorial plane intersects with the projection ball is called the basic circle (circle NESW in figure 1). Mark the scales on the basic circle, it is used to determine the extended orientation of planes and lines in three dimensional space when making spherical projection.

\subsection{Stereographic Projection}

Stereographic projection is a method of converting spherical projection into plane projection. The transformation method is that a series of radials are projected on the sphere from one of the upper polar emitter point and lower polar emitter point, the intersection points of the radials with the equatorial plane are the projection of the straight lines pass through the center of the projection ball: Straight lines through the centre of the projection ball: the stereographic projection of the plumb line is the center of the basic circle(point $\mathrm{O}$ in figure 1), the stereographic projection of the horizontal lines ( $\mathrm{SN}$ and $\mathrm{EW}$ in figure 1) are the two intersection points $(\mathrm{S}, \mathrm{N}$, and $\mathrm{E}, \mathrm{W}$ in figure 1)with the basic circle, the stereographic projection of the oblique lines ( $A B$ in figure 1 ) is a point ( $\mathrm{A}^{\prime}$ in figure 1$)$ in the basic circle. The planes pass through the centre of the projection ball, the stereographic projection of the vertical plane (SPNF in figure 1) is a diameter line ( $\mathrm{SN}$ in figure 1) of the basic circle, the stereographic projection of the horizontal plane (ESWN) overlaps with the basic circle (ESWN), the stereographic projection of the oblique plane (ASBN) is an arc (C) of the basic circle diameter (SN) as chord. The planes not pass through the centre of the projection ball, the stereographic projection of the vertical plane is the same two small arcs intersect with the basic circle which are symmetrical, the stereographic projection of the horizontal plane is the concentric circle of the basic circle, the stereographic projection of an oblique plane is a small circle (circle $\mathrm{C} 1$ in figure 1 ) within the basic circle, or could be the arc intersect with basic circle (arc C2 in figure 1).

\section{Method of Improved Stereographic Projection}

The traditional method of stereographic projection always applies in geoscience to solve the small scale of the earth problems, and the geographical location of the research point was not considered. In the study of large scale or geographic coordinates related to the tectonic problems, the traditional stereographic projection method is not applicable. The movement of lithospheric plates on the earth surface is large scale tectonic problem and involves geographic coordinates. Therefore, when we applied the method of stereographic projection to study plate motion trajectory, we improved the traditional method of stereographic projection. In order to distinguish it from the traditional method, which called it "improved stereographic projection". Furthermore, the principle of the traditional stereographic projection method is applicable to the "improved stereographic projection". Based on the standard Wulff's net, the eastern and western hemispheres stereographic projection and the southern and northern hemispheres stereographic projection were established. 

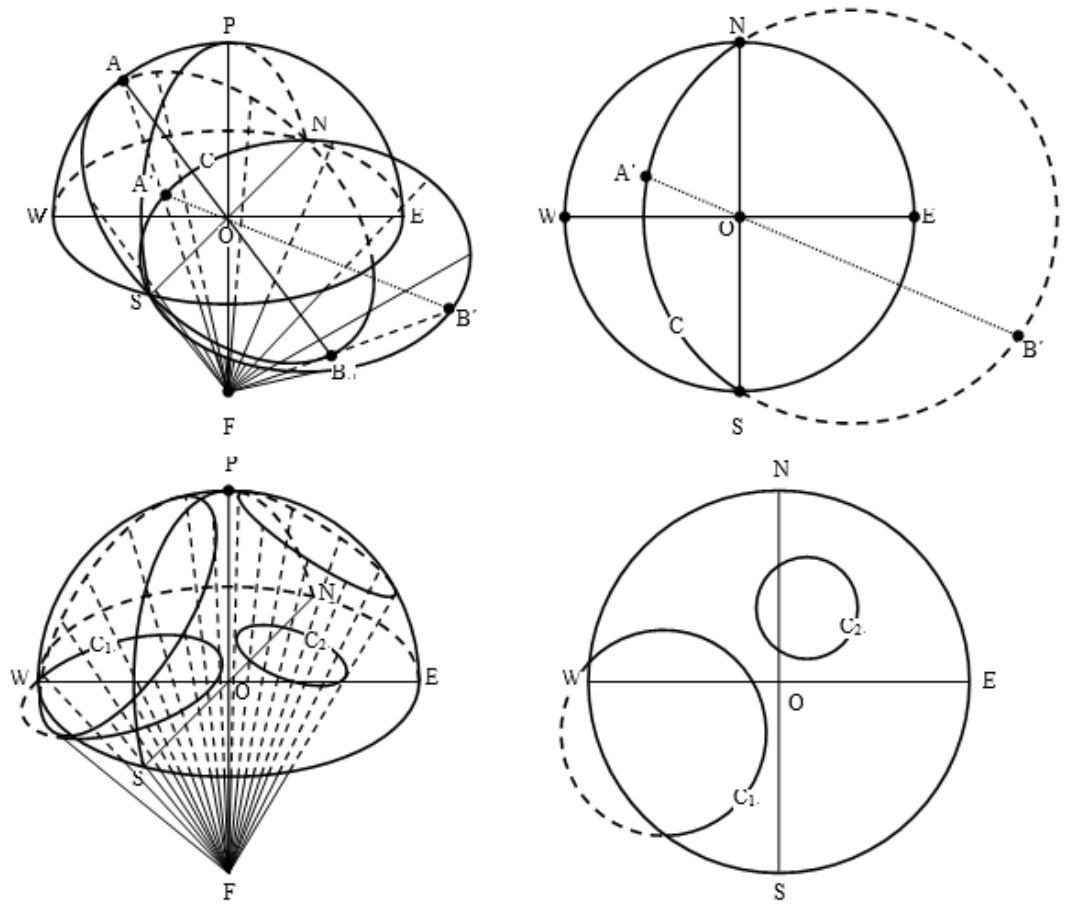

Figure 1. Transformation between spherical projection and stereographic projection.

\subsection{Stereographic Projection of Eastern and Western Hemispheres}

The stereographic projection of eastern and western hemispheres is taking the earth as the projection ball, the plane where the axis is located as the equatorial plane, and the center of the earth as the center of the projection ball. The equatorial plane determined in the projection is also determined by the geographic meridian plane. The east-west diameter line and the north-south diameter determine the basic circle of the improved stereographic projection. The upper and lower polar emitter points are the intersection points between the earth axis with the projection ball, the scales on the basic circle represent the latitude and do not indicate the orientation.

\subsection{Stereographic Projection of Southern and Northern Hemispheres}

The stereographic projection of southern and northern hemispheres is taking the earth as the projection ball, the plane where the equator is located as the equatorial plane, and the earth center as the center of the projection ball. The east-west diameter line of the basic circle is the straight line of the equator, the north-south diameter line of the basic circle is the intersection between the earth axis plane and the equatorial plane, these two perpendicular intersection lines determine the basic circle of the improved stereographic projection. The upper and lower polar emitter points are geographic north and south poles, the scales on the basic circle represent the longitude and do not indicate the orientation. 


\subsection{Stereographic Projection of Determining Plate Motion Trajectory}

The motion of the lithospheric plates on the earth surface is not simple horizontal movement, but is the spherical motion of rotating around the earth center, this is consistent with Euler's rotation theorem. In general, tectonic stress field is generated when tectonic activity occurs during plate motion. In order to understand the tectonic stress field, that is necessary to know the paleomagnetic data of three adjacent geological ages of the plate. According to the three adjacent geological ages paleomagnetic data of the plate, we can get two Euler poles within the spherical great circle. In the process of plate motion, the research point (a point on the target plate) moves from point $\mathrm{A}$ to point $\mathrm{B}$ and then to point $\mathrm{C}$, the geological ages of $\mathrm{A}, \mathrm{B}$ and $\mathrm{C}$ are three adjacent geological eras, and the chronological order is old to new (figure 2). If the magnetic declination $\mathrm{D}$ and the paleolatitude $\omega 2$ of point $\mathrm{B}$ are known, then according to the magnetic declination direction and the magnitude, paleolatitude of point $\mathrm{A}$ and $\mathrm{C}$ in the geological era can be determined. According to the angular distance is $90^{\circ}-\omega$, we can get the paleomagnetic poles location of the corresponding geological age. So, the position of the paleomagnetic pole, following the movement route of the geological age is: from NO to NA, then, from NA to NB (figure 2). Based on the paleomagnetic pole data of the motion from point $\mathrm{A}$ to $\mathrm{B}$, a great circular arc SNAC can be drawn, similarly, the great circular arc SNAB of the motion from point B to $\mathrm{C}$ can be drawn. The intersection point (point $\mathrm{O}$ in figure 2) of these two great circular arcs is the Euler pole of this period of plate movement, therefore, the drift motion of the research point on the plate is determined.

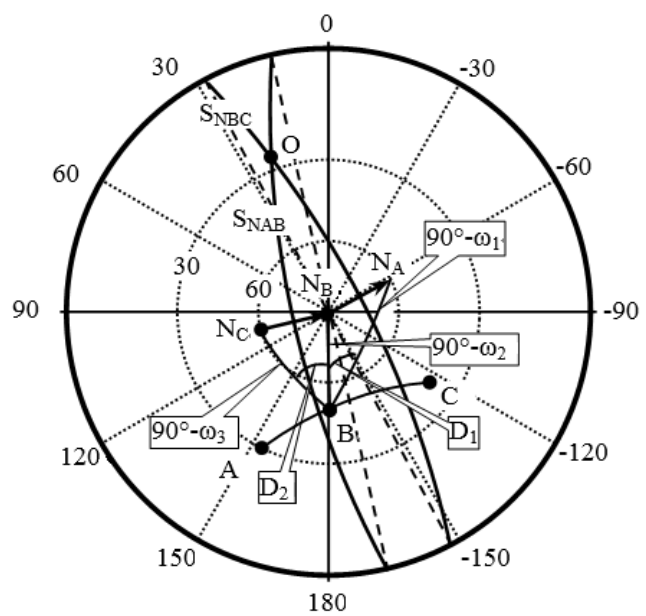

Figure 2. Stereographic projection of lithospheric plate three adjacent ages paleomagnetic data.

\section{Results}

For this study, we used to result from an updated global data set carefully reprocessed by Klootwijk et al. This data includes the paleomagnetic information of the Indian plate in geological ages from Cretaceous (table 1). 
Table 1. Paleomagnetic data of the Indian plate (the reference center point: $79^{\circ} \mathrm{E}, 21^{\circ} \mathrm{N}$ ).

\begin{tabular}{lllll}
\hline Geological age (Ma) & $\mathbf{N}$ & $\boldsymbol{\alpha}_{95}$ & $\boldsymbol{D}, \boldsymbol{\omega}\left(^{\circ}\right)$ & $\boldsymbol{\psi}, \boldsymbol{\lambda}\left(^{\circ}\right)$ \\
\hline $\mathrm{N}(5-22.5)$ & 1 & 2.3 & $353,11.5$ & $114.5,-78.5$ \\
$\mathrm{E}_{3}(25-35)$ & 1 & 3.5 & $345.1,10.9$ & $136,-72.5$ \\
$\mathrm{E}_{2}(45-55)$ & 1 & 9 & $158.7,3.9$ & $121.5,-57.5$ \\
$\mathrm{E}_{1}(60-65)$ & 4 & 16.6 & $334.4,-29.2$ & $286.1,34.1$ \\
$\mathrm{~K}_{2}(66-100)$ & 17 & 5 & $318,-35.1$ & $294.7,21.4$ \\
$\mathrm{~K}_{1}(101-137)$ & 7 & 3.6 & $305,-43.8$ & $295.7,7.9$ \\
\hline
\end{tabular}

Note: N-number of stratigraphic levels; $D$-declination angle; $\alpha_{95}$-radius of reliability circle for probability of $0.95 ; \omega$-palaeolatitude; $\psi$-geomagnetic pole longitude; $\lambda$-geomagnetic pole latitude.

We used the paleomagnetic data to study the motion trajectory of the Indian plate. Firstly, we projected the central reference point geographic coordinates $\left(79^{\circ} \mathrm{E}, 21^{\circ} \mathrm{N}\right)$ of the Indian plate nowadays into the northern hemisphere stereographic projection net. After that, we projected the seven geomagnetic pole coordinates (six in table 1 and the nowadays coordinate of the Indian plate-1 Nowadays $\left(0^{\circ}, 90^{\circ}\right), 2 \mathrm{~N}\left(114.5^{\circ},-78.5^{\circ}\right), 3$ $\left.\mathrm{E} 3\left(136^{\circ},-72.5^{\circ}\right), 4 \mathrm{E} 2(121.5,-57.5), 5 \mathrm{E} 1(286.1,34.1), 6 \mathrm{~K} 2(66-100), 7 \mathrm{~K} 1(295.7,7.9)\right)$ since the early cretaceous into the northern hemisphere stereographic projection net. It should be noted that the latitude of the 2N, 3E3 and 4E2 geomagnetic poles coordinates are negative, that's because these three paleomagnetic poles are in the southern hemisphere. Due to the latitude lines of the southern hemisphere and the northern hemisphere are symmetrical, so, after the processing of these geomagnetic poles coordinates--latitude positively, we projected the seven processed geomagnetic poles coordinates into the northern hemisphere stereographic projection net (figure 3a). After we connected the seven points, we can get the geomagnetic pole migration path of the Indian plate from the early cretaceous. According to the paleomagnetic data of three adjacent geological time can determine an Euler pole, and can determine a motion direction, we chose three adjacent geological ages as a movement stage. The movement of the Indian plate since the early cretaceous can be divided into three stages: the oldest stage, $7(\mathrm{~K} 1) \rightarrow 6(\mathrm{~K} 2) \rightarrow 5(\mathrm{E} 1)$, the drift motion time is $72 \mathrm{Ma}$; the middle stage, $5(\mathrm{E} 1)$ $\rightarrow 4(\mathrm{E} 2) \rightarrow 3(\mathrm{E} 3)$, the drift motion time is $30 \mathrm{Ma}$; the latest stage, $3(\mathrm{E} 3) \rightarrow 2(\mathrm{~N}) \rightarrow$ 1 (Nowadays), the drift motion time is $35 \mathrm{Ma}$. According to the migration path of the geomagnetic pole migration, the motion trajectory of the Indian plate at three stages can be determined by use of stereographic projection analysis. The three adjacent geological age coordinate points of each stage are on the same circle, and the motion trajectory is moving along the circle (figure $3 b$ ), the trajectory of each motion stage is a circular arc. 

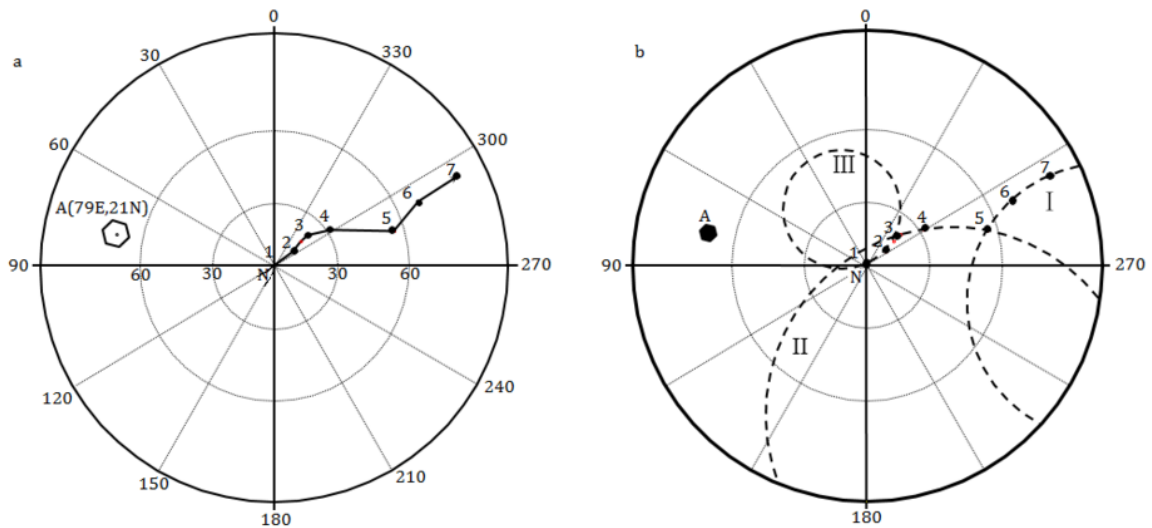

Figure 3. Stereographic projection analysis of Indian plate every period motion.

The latest stage, from $35 \mathrm{Ma}$ to the present, we projected 1Nowdays $\left(0^{\circ}, 90^{\circ}\right), 2 \mathrm{~N}$ $\left(114.5^{\circ},-78.5^{\circ}\right), 3 \mathrm{E} 3\left(136^{\circ},-72.5^{\circ}\right)$ on the northern hemisphere stereographic projection net, then, we can get the result of Indian plate the latest stage movement trajectory. In this stage, the movement end point is A which locate at the central point of the Indian plate nowadays, its geographic coordinate is $79^{\circ} \mathrm{E}, 21^{\circ} \mathrm{N}$.

\section{Conclusions}

In this paper, the traditional method of stereographic projection had been improved and transformed, and we mentioned a new method to quantify the motion trajectory of lithosphere plate-improved stereographic projection method.

Based on the improved stereographic projection analysis by using paleomagnetic data of the Indian plate, we divided the movement of the Indian plate into three stages: the oldest stage, from $137 \mathrm{Ma}$ to $65 \mathrm{Ma}$, the motion trajectory is from $73^{\circ} \mathrm{E}, 41^{\circ} \mathrm{S}$ to $65^{\circ} \mathrm{E}, 28^{\circ} \mathrm{S}$, the motion direction is NNW; the middle stage, from $65 \mathrm{Ma} \sim 35 \mathrm{Ma}$, the motion trajectory is from $65^{\circ} \mathrm{E}, 28^{\circ} \mathrm{S}$ to $43^{\circ} \mathrm{E}, 10^{\circ} \mathrm{N}$, the motion direction is $\mathrm{NW}$; the latest stage, from $35 \mathrm{Ma}$ to the present, the motion trajectory is from $43^{\circ} \mathrm{E}, 10^{\circ} \mathrm{N}$ to $79^{\circ} \mathrm{E}, 21^{\circ} \mathrm{N}$, the motion direction is NE. The motion direction of Indian plate changed greatly at $35 \mathrm{Ma}$, it may be due to restriction and influence of other plates, such as Eurasian plate.

Using the paleomagnetic data of target lithospheric plate and the improved stereographic projection method, the lithospheric plate motion trajectory can be interpreted quantitatively. This method has an extensive application prospect, but we must ensure the accuracy of the paleomagnetic data to ensure the accuracy of the improved stereographic projection analysis. The premise of popularizing this method is accurate paleomagnetic data, and time interval of paleomagnetic samples are small enough. 


\section{Acknowledgments}

The authors thank for the support from the Education Department of Guizhou Province Youth Science and Technology Talent Growth Project (Qianjiaohe KY Zi [2018]380 and [2018]383), and High level Talents Project of Liupanshui Normal University (LPSSYKYJJ 201407). Mining Engineering First class discipline (Group) construction in Guizhou (QJ XKTJ [2020]23). Guizhou Province roadway roof visualization intelligent detection technology innovation talent team (Qiankehe platform talent [2019] $5620)$.

\section{References}

[1] Turcotte D L and Schubert G 2002 Plate Teoctonics, Geodynamics 2nd ed (Cambridge University Press) pp 1-21.

[2] David G 2010 The distinction between geomagnetic excursions and reversals Geophysical Journal International (1) F1-F4.

[3] Hoffman K A 2003 Evidence for Weak Geomagnetic Field Intensity Prior to the Cretaceous Normal Superchron (Institute of Geology and Geophysics Chinese Academy of Sciences) 13.

[4] Jacobs J A 1995 The earth's magnetic field and reversals Endeavour 19 (4) 166-171.

[5] Besse J and Courtillot V 1991 Revised and synthetic apparent polar wander paths of the African, Eurasian, North American and Indian plates and true polar wander paths since 200Ma Journal of Geophysical Research Solid Earth 96 (B3) 4029-4050.

[6] Cande S C and Kent D V 1995 Revised calibration of the geomagnetic polarity timescale for the late Cretaceousan Cenozoic Journal of Geophysical Research 100 (B4) 6093-6095.

[7] Li Y P, Zhang Z K and Mcwilliams M 2013 Mesozoic paleomagnetic results of the Tarim craton: Tertiary relative motion between China and Siberia Geophysical Research Letters 15 (3) 217-220.

[8] Cogné J P, Chen Y and Courtillot V 1995 A paleomagnetic study of Mesozoic sediments from the Junggar and Turfan basins, northwestern China Earth Planet Science Letters 133 (3-4) 353-366.

[9] Gilder S A, Zhao X X and Coe R S 1996 Paleomagnetism and tectonics of the southern Tarim Basin, northwestern China Journal of Geophysical Research Solid Earth 101 (B10) 22015-22031. 